\title{
REMARKS ON A THEOREM OF E. J. MCSHANE ${ }^{1}$
}

\section{B. J. PETTIS}

In a recent paper E. J. McShane $[3]^{2}$ has given a theorem which is the common core of a variety of results about Baire sets, Baire functions, and convex sets in topological spaces including groups and linear spaces. In general terms his theorem states that if $\mathcal{F}$ is a family of open maps defined in one topological space $X_{1}$ into another, $X_{2}$, the total image $\mathcal{F}(S)$ of a second category Baire set $S$ in $X_{1}$ has, under certain conditions on $\mathcal{F}$ and $S$, a nonvacuous interior. The point of these remarks is to show that his argument yields a theorem for a larger class than the second category Baire sets. From this there follow slightly stronger and more specific versions of some of his results, including his principal theorem, as well as a proof that if $S$ is a subset of a weak sort of topological group and $S$ contains a second category Baire set, then the identity element lies in the interior of both $S^{-1} S$ and $S S^{-1}$. There is also at the end an extension of Zorn's theorem on the structure of certain semigroups.

In a topological space $X$ let the closure and interior of a set $E$ be denoted by $E^{*}$ and $E^{0}$ and the null set by $\Lambda$. For any set $S$ let $I(S)$ $=\cup[G \mid G$ open, $G \cap S$ is first category $]$ and $I I(S)=X-I(S)$, and let $I I I(S)$ be the open set $I I(S)^{\circ} \cap I(X-S)$. By a fundamental theorem of Banach [2], $S \cap I(S)^{*}$ is first category and hence $S$ is second category if and only if $I I(S)^{0} \neq \Lambda$. From these we note that if $N$ is a non-null open subset of $I I I(S)$, then $N-S$ is in the first category set $(X-S) \cap I(X-S)$, and $N \cap S$ cannot be first category since $N$ is non-null open and disjoint with $I(S)$. This gives us the following lemma.

Lemma 1. For any non-null open subset $N$ of $\operatorname{III}(S)$, the sets $N-S$ and $N \cap S$ are first and second category respectively.

We recall that $S$ is defined to be a Baire set in $X$ if $(S-G) \cup(G-S)$ is first category for some open set $G$; an equivalent condition is $I I(S)^{0} \subset I(X-S)$, or $I I(S)^{0}=I I I(S)$.

DEFINITION. $\mathfrak{B}(X)$ is the family of all second category Baire sets in $X$. $A$ set $S$ is in $\mathfrak{A}(X)$ if and only if $S$ contains an element of $\mathfrak{B}(X)$.

Received by the editors December 15, 1949 and, in revised form, January 27, 1950.

${ }^{1}$ This paper was written under Contract N7-onr-434, Task Order III, Navy Department (The Office of Naval Research).

${ }^{2}$ Numbers in brackets refer to the references at the end of the paper. 
Any member $S$ of $\mathfrak{B}(X)$ is characterized by $I(X-S) \supset I I(S)^{0} \neq \Lambda$ or by $I I I(S)=I I(S)^{0} \neq \Lambda$. A characterization of the class $\mathfrak{A}(X)$, which is generally larger (for example, when $X$ is the reals), is given by the following lemma.

Lemma 2. $S \in \mathfrak{A}(X)$ is equivalent to each of these: (1) $I I I(S) \neq \Lambda,(2)$ there is a non-null open set $N$ such that $N-S$ is first category and $N \cap S$ is second category.

If $S \in \mathfrak{A}(X)$, let $B \in \mathfrak{B}(X)$ with $B \subset S$. The latter implies $I I(S)^{0}$ $\supset I I(B)^{0}$ and $I(X-S) \supset I(X-B)$ and hence $I I I(S) \supset I I I(B) \neq \Lambda$. When (1) holds, the set $N=I I I(S)$ satisfies (2) by Lemma 1 above. When (2) is true, let $B=N \cap S$. Then $B$ is second category and differs from the open set $N$ only on the first category set $N-S$; thus $B \in \mathfrak{B}(X)$.

Now let $X_{1}$ and $X_{2}$ be topological spaces and $\mathcal{F}=[f]$ a non-null family of functions defined in $X_{1}$ to $X_{2}$ where the domain of definition $D_{f}$ of each $f$ is open in $X_{1}$ and each $f$ is open on its domain to $X_{2}$, that is, maps open sets into open sets. For any sets $E_{1} \subset X_{1}$ and $E_{2} \subset X_{2}$ we shall write $\mathcal{F}\left(E_{1}\right)$ for the set $U\left[f\left(E_{1} \cap D_{f}\right) \mid f \in F\right]$ and $\mathcal{F}^{-1}\left(E_{2}\right)$ for the set of all $x \in X_{1}$ such that $f(x)$ is defined and in $E_{2}$ for some $f \in \mathcal{F}$.

For any pair of sets $S_{0}$ and $N$ in $X_{1}$ consider these conditions on $S_{0}, N$, and $\mathcal{F}$ : (i) $S_{0} \neq \Lambda$ and $S_{0} \subset D_{f}$ for each $f \in \mathcal{F}$; (ii) $N$ is open and $N \supset S_{0}$; (iii) $f\left(N \cap D_{f}\right) \subset \mathcal{F}\left(S_{0}\right)$ for each $f \in \mathcal{F}$. We note that (iii) is equivalent to the following: (iii') $y \in \mathcal{F}(N)$ implies $S_{0} \cap \mathcal{F}^{-1}(y) \neq \Lambda$. The essential proposition in McShane's theorem can be stated as follows.

Lemma 3. When $\mathcal{F}, S_{0}$, and $N$ satisfy (i), (ii), and (iii), $\mathcal{F}\left(S_{0}\right)$ is non-null and open.

For (following McShane) if $U=\bigcup_{f} f\left(N \cap D_{f}\right)$, then from the original assumptions on $\mathcal{F}$ clearly $U$ is open in $X_{2}$, and $U \subset \mathcal{F}\left(S_{0}\right)$ from (iii). By (i) and (ii) on the other hand, $S_{0} \subset D_{f} \cap N$ for each $f$ and hence $\mathcal{F}\left(S_{0}\right) \subset U$. And $\mathcal{F}\left(S_{0}\right) \neq \Lambda$ since $S_{0}$ and $\mathcal{F}$ are nonvacuous and $S_{0} \subset D_{f}$ for all $f$.

Now suppose these are true for two sets $N$ and $S$ in $X_{1}:\left(\mathrm{i}^{\prime}\right)$ $\Lambda \neq N \cap S \subset D_{f}$ for all $f$ in $\mathcal{F}$; (ii') $N$ is open. If we set $S_{0}=N \cap S$ clearly (i) and (ii) hold; hence we have the following lemma.

Lemma 4. If $\mathcal{F}, S$, and $N$ satisfy (i') and (ii') and also either (iii) or (iii') with $S_{0}=N \cap S$, then $\mathcal{F}(N \cap S)$ is a non-null open set.

Obviously (iii') is true for $S_{0}=N \cap S$ if $N \cap S \cap \mathcal{F}^{-1}(y)$ is second 
category for each $y \in \mathcal{F}(N)$; and the latter clearly holds whenever $N-S$ is first category and the following is true: (iii") $y \in \mathcal{F}(N)$ implies $N \cap \mathcal{F}^{-1}(y)$ is second category. Lemma 4 now gives us the following lemma.

LEMMA 5. $\mathcal{F}(N \cap S)$ is non-null and open whenever $\mathcal{F}, S$, and $N$ satisfy (i'), (ii'), and (iii') and $N-S$ is first category.

From Lemmas 1 and 5 we have immediately the following theorem.

THEOREM. $\mathcal{F}(N \cap S)$ is non-null and open provided that $N$ is a nonnull open subset of $\operatorname{III}(S)$, that $D_{f} \supset S \cap N$ for each $f$ in $\mathcal{F}$, and that (iii") is satisfied.

In view of Lemma 2 the theorem is concerned with and only with elements $S$ of $\mathfrak{A}\left(X_{1}\right)$. When $S$ is in the more restricted class $\mathfrak{B}\left(X_{1}\right)$, that is, when $I I I(S)=I I(S)^{0} \neq \Lambda$, the following slightly sharper version of McShane's theorem results on taking $N$ to be $I I(S)^{0}$.

Corollary 1. If $S \in \mathfrak{B}\left(X_{1}\right)$, if $D_{f} \supset S \cap I I(S)^{0}$ for each $f$ in $\mathcal{F}$, and if $I I(S)^{0} \cap \mathcal{F}^{-1}(y)$ is second category for each $y \in \mathcal{F}(N)$, then $\mathcal{F}(S)$ $=\mathcal{F}\left(S \cap I I(S)^{0}\right) \cup \mathcal{F}\left(S \cap I(S)^{*}\right)$ where $\mathcal{F}\left(S \cap I I(S)^{0}\right)$ is non-null and open in $X_{2}$ and $S \cap I(S) *$ is first category in $X_{1}$.

Now let $X$ be a group having a topology in which $x y$ is continuous in each variable and let $e$ be the identity element. We recall the following properties of the function $I I(E)$ in any topological space [2, pp. 46-47]: $(\alpha) I I(E)$ is always closed, $I I(I I(E))=I I(E)$, and $I I(E) \subset I I(F)$ when $E \subset F ;(\beta)\left(I I(E)^{0}\right)^{*}=I I(E) \subset E^{*} ;(\gamma)$ for any open set $G, I I\left(G \cap E \cap I I(E)^{0}\right) \supset G \cap I I(E)$; and $(\delta)$ for any homeomorphism $\phi$ in $X, I I(\phi(E))=\phi(I I(E))$.

Corollary 2. Let $R$ be second category in $X$ and $S \in \mathfrak{A}(X)$. Suppose $G$ and $H$ are open and $G \cap I I(R) \neq \Lambda \neq H \cap I I I(S)$. If we set $A=G \cap R$ $\cap I I(R)$ and $B=H \cap S \cap I I I(S)$, it follows that $A^{-1} B$ and $B A^{-1}$ are non-null open subsets of $R^{-1} S$ and $S R^{-1}$ respectively.

For each $a \in A$ define $f_{a}(x)=a^{-1} x$ for all $x$ in the non-null open set $N=H \cap I I I(S)$ and set $\mathcal{F}=\left[f_{a}\right]$. Since $G \cap I I(R) \neq \Lambda$ it follows from $(\gamma)$ above that $A \neq \Lambda$. Thus $\mathcal{F}$ is non-null, each $f_{a}$ is open on its open domain, and $D_{f_{a}} \supset S \cap N=B$ for each $f_{a}$. If it is shown that $N \cap \mathcal{F}^{-1}(y)$ is second category whenever $y \in \mathcal{F}(N)$, the theorem is applicable and $\mathcal{F}(N \cap S)=\mathcal{F}(B)=A^{-1} B$ is a non-null open subset of $R^{-1} S$. If $y \in \mathcal{F}(N)$, then $y=a^{-1} x$ for some $a \in A$ and $x \in N$, and $\mathcal{F}^{-1}(y)=A a^{-1} x$. Hence $I I\left(\mathcal{F}^{-1}(y)\right)=I I\left(A a^{-1} x\right)=I I(A) a^{-1} x$ by $(\delta)$ above; since $I I(A) \supset A$ by $(\gamma)$, it follows that $I I\left(\mathcal{F}^{-1}(y)\right) \ni x$. Then, since $N \ni x$ and is open, 
$N \cap \mathcal{F}^{-1}(y)$ must be second category. A similar proof, setting $f_{a}(x)$ $=x a^{-1}$, establishes the theorem's other assertion.

Taking $G=H=X$ we have the following corollaries.

Corollary 3. Let $S \in \mathfrak{A}(X)$. If $R$ is second category then $[R$ $\cap I I(R)]^{-1}[S \cap I I I(S)]$ and $[S \cap I I I(S)][R \cap I I(R)]^{-1}$ are non-null open subsets of $R^{-1} S$ and $S R^{-1}$ respectively.

Corollary 4. If $S \in \mathfrak{A}(X)$ and $R^{-1}$ is second category the sets $R S$ and $S R$ have non-null interiors.

Corollary 5. When $S \in \mathfrak{A}(X)$ it follows that $e \in\left(S^{-1} S\right)^{0} \cap\left(S S^{-1}\right)^{0}$.

CoRollary 6. If $S$ is a subgroup and $S \in \mathfrak{A}(X)$, then $S=S^{0}$ and hence, since $S$ is a subgroup, $S=S^{*}$.

Corollaries 4 and 6 are slight extensions of results of McShane and Banach [3]. Corollary 5 for a more restricted $X$ is in another paper [4].

Corollary 7. Suppose $E^{-1}$ is second category in $X$ whenever $E$ is second category. If $R$ is second category and $S \in \mathfrak{A}(X)$, then

$$
\left[R \cap I I\left(R^{-1}\right)^{-1}\right][S \cap I I I(S)] \text { and }[S \cap I I I(S)]\left[R \cap I I\left(R^{-1}\right)^{-1}\right]
$$

are non-null open subsets of $R S$ and $S R$ respectively, and

$$
I I(R) I I I(S)^{*} \subset\left((R S)^{0}\right)^{*} \text { and } I I I(S)^{*} I I(R) \subset\left((S R)^{0}\right)^{*} .
$$

Conclusion (1) results immediately from Corollary 3 since $R^{-1}$ is second category. To establish (2) let $C=R \cap I I\left(R^{-1}\right)^{-1}$ and $D=S$ $\cap I I I(S)$, and note that (1) implies that $C^{*} D^{*} C(C D)^{*} \subset\left((R S)^{0}\right)^{*}$ and $D^{*} C^{*} \subset\left((S R)^{0}\right)^{*}$. It is thus sufficient to show that $I I(R) \subset C^{*}$ and $I I I(S) \subset D^{*}$. The latter follows from $(\gamma)$ above; for on setting $G=I(X-S)$ and $E=S$ therein we have $I I(D) \supset I I I(S)$ and hence $D^{*} \supset I I I(S)$. For the former, consider any open set $N$ intersecting $I I(R)$. The set $N \cap R$ is second category and hence $(N \cap R)^{-1}$ is a second category subset of $R^{-1}$. From this it follows that $(N \cap R)^{-1}$ $\cap I I\left(R^{-1}\right) \neq \Lambda$, for otherwise $(N \cap R)^{-1}$ is in the first category set $R^{-1} \cap I\left(R^{-1}\right)$. Taking inverses we have $\Lambda \neq N \cap R \cap I I\left(R^{-1}\right)^{-1}=N$ $\cap C$, proving that $I I(R) \subset C^{*}$.

When $S \in \mathfrak{B}(X)$ the terms $I I I(S)$ and $I I I(S)^{*}$ in Corollary 7 can be replaced by $I I(S)^{0}$ and $I I(S)$, and hence in particular $I I(S)^{2}$ $\subset\left(\left(S^{2}\right)^{0}\right)^{*}$. If also $S$ is a semigroup, that is, $S^{2} \subset S$, then $I I(S)^{2} \subset\left(S^{0}\right)^{*}$. It may also be remarked that the first assumption in Corollary 7 is weaker than that of assuming $x^{-1}$ to be continuous in $x$, as is shown by the reals with intervals $a \leqq x<b$ as neighborhoods. 
LEMma 6. For any sets $R$ and $S$ in $X$ let $\Gamma(R, S)=I I(R) S^{*} \cup R^{*} I I(S)$ $\cup I I(R) I I\left(S^{*}\right) \cup I I\left(R^{*}\right) I I(S)$ and $\Delta\left(R, S^{*}=I I(R) S^{*} \cup R^{*} I I(S)\right.$ $\cup I I(R) I I(S)$. Then $(R S)^{*} \supset I I(R S) \supset \Gamma(R, S) \supset \Delta(R, S) \supset I I(R) I I(S)$.

For any $s \in S$ and $r \in R$ we have $I I(R) s=I I(R s) \subset I I(R S)$ and $r I I(S)=I I(r S) \subset I I(R S)$, so that $I I(R) S \cup R I I(S) \subset I I(R S)$. Since $I I(R S)$ is closed and $A^{*} B^{*} C(A B)^{*}$ for any $A$ and $B$, it follows that $I I(R) S^{*} \cup R^{*} I I(S) \subset I I(R S)$. Moreover $I I(I I(E))=I I(E)$ for any $E$; hence $I I(R S)=I I(I I(R S)) \supset I I\left(I I(R) S^{*}\right) \cup I I\left(R^{*} I I(S)\right)$. But from what has already been shown we have $I I\left(I I(R) S^{*}\right) \supset I I(I I(R))\left(S^{*}\right)^{*}$ $\cup I I(R)^{*} I I\left(S^{*}\right)=I I(R) S^{*} \cup I I(R) I I\left(S^{*}\right)$, and similarly $I I\left(R^{*} I I(S)\right)$ $\supset I I\left(R^{*}\right) I I(S) \cup R^{*} I I(S)$. Thus $I I(R S) \supset \Gamma(R, S)$. The rest is obvious.

From this it is clear that $I I\left(S^{2}\right) \supset \Gamma(S, S) \supset \Delta(S, S) \supset I I(S)^{2}$ for any $S$. Another consequence is the following corollary.

Corollary 8. Suppose $E^{-1}$ is second category whenever $E$ is second category. If $R$ is second category, $S \in \mathfrak{B}(X)$, and $S \supset R S \cup S R$, then

$$
\left(S^{0}\right)^{*} \supset I I(R)[\Gamma(R, S) \cup \Gamma(S, R)] \cup[\Gamma(R, S) \cup \Gamma(S, R)] I I(R) .
$$

Clearly $\left(S^{0}\right)^{*} \supset\left((R S)^{0}\right)^{*}$, and from a remark after Corollary 7, $\left((R S)^{0}\right)^{*} \supset I I(R) I I(S)$. At the same time, obviously $I I(S) \supset I I(R S)$ $\cup I I(S R)$, where $I I(R S) \supset \Gamma(R, S)$ and $I I(S R) \supset \Gamma(S, R)$ by Lemma 6. Thus $\left(S^{0}\right)^{*} \supset I I(R)[\Gamma(R, S) \cup \Gamma(S, R)]$. Similarly, $\left(S^{0}\right)^{*} \supset\left((S R)^{0}\right)^{*}$ $\supset[\Gamma(S, R) \cup \Gamma(R, S)] I I(R)$.

When $S_{1}$ is a semigroup, this has obvious consequences, first when $S_{1} \in \mathfrak{B}(X)$ and $R=S=S_{1}$ and second when $S_{1}$ is in $\mathfrak{B}(X)$ and $R=S_{1}^{-1}$ and $S=X-S_{1}$. These together imply Corollary 5 of [3].

Lemma 7. For any subset $S$ of a topological space $X$ these conditions are equivalent: (1) $S$ is a Baire set, $S \subset I I(S)$, and $X-S \subset I I(X-S)$; (2) the equalities (i) $S^{0}=I I(S)^{0}=\left(S^{*}\right)^{0}=I(X-S)$ and (ii) $S^{*}=I I(S)$ $=\left(S^{0}\right)^{*}=I(X-S)^{*}$ are true; (3) equalities (i) and (ii) hold when $S$ and $X-S$ are interchanged.

Taking complements in (i) and (ii) yields (ii) and (i) with $S$ and $X-S$ interchanged; thus (2) and (3) are equivalent. Concerning (1) and (2) we note that by $(\beta)$ above $S \subset I I(S)$ is equivalent to (4) $S^{*}=I I(S)$, that $X-S \subset I I(X-S)$ is equivalent to $(X-S)^{*}$ $=I I(X-S)$, that is, to (5) $S^{0}=I(X-S)$, and recall that $S$ is a Baire set if and only if (6) $I I(S)^{\circ} \subset I(X-S)$. Obviously (2) now implies (1). Conversely, (1) implies (4), (5), and (6), where (6) implies (7) $I I(S) \subset I(X-S)^{*}$ since $I I(S)=\left(I I(S)^{0}\right)^{*}$. From (4), (7), and (5) we have $S^{*}=I I(S) \subset I(X-S)^{*}=\left(S^{0}\right)^{*}$; since $\left(S^{0}\right)^{*} \subset S^{*}$, (ii) follows. 
Taking interiors in (ii) yields $\left(S^{*}\right)^{0}=I I(S)^{0}=\left(I(X-S)^{*}\right)^{0}$; since $\left(I(X-S)^{*}\right)^{0}=\left((X-I I(X-S))^{*}\right)^{0}=\left(X-I I(X-S)^{0}\right)^{0}=X-(I I(X$ $\left.-S)^{0}\right)^{*}=X-I I(X-S)=I(X-S)$ and (5) holds, (i) now follows also.

Lemmas 6 and 7, which are independent of the other lemmas and corollaries, provide the following mild extension of Zorn's theorem on the structure of a semigroup $S$ when $S$ is a Baire set such that $S$ and $S^{-1}$ are second category at $e$ [1, pp. 157-158; 3, Corollary 6].

THEOREM. Suppose $S$ is a Baire set and $S \supset R S$ or $S \supset S R$ for some $R$ such that $I I(R)^{m} \cap I I\left(R^{-1}\right)^{n} \ni e$ for some $m$ and $n \geqq 1$. Then (2) and (3) of Lemma 7 are true.

Suppose $S \supset R S$. From Lemma 6 and the definition of $\Delta(R, S), S^{*}$ $\supset I I(S) \supset I I(R S) \supset \Delta(R, S) \supset I I(R) S^{*}$, so that $S^{*} \supset I I(S) \supset I I(R) S^{*}$. Multiplying by $I I(R)^{k}$ we have $I I(R)^{k} S^{*} \supset I I(R)^{k+1} S^{*}$, and hence $S^{*} \supset I I(S) \supset I I(R)^{k} S^{*}$ for any $k \geqq 1$. When $e \in I I(R)^{m}$, it is then clear that $S^{*} \supset I I(S) \supset S^{*}$, or $S^{*}=I I(S)$. Since $S \supset R S$ implies $X-S$ $\supset R^{-1}(X-S)$, we also have $(X-S)^{*}=I I(X-S)$ in case $e \in I I\left(R^{-1}\right)^{n}$ for some $n \geqq 1$. A similar proof applies when $S \supset S R$. Thus our hypotheses here imply (1) of Lemma 7, and the present conclusion follows.

\section{REFERENCES}

1. E. Hille, Functional analysis and semi-groups, Amer. Math. Soc. Colloquium Publications, vol. 31, New York, 1948.

2. C. Kuratowski, Topologie I, Monografie Matematyczne III, Warsaw-Lw6w, 1933.

3. E. J. McShane, Images of sets satisfying the condition of Baire, Ann. of Math. vol. 51 (1950) pp. 380-386.

4. B. J. Pettis, On continuity and openness of homomorphisms in topological groups, Ann. of Math. vol. 52 (1950) pp. 293-308.

TUlane University and

Princeton University 